TITLE:

\title{
Slow viscous flows in micropolar fluids
}

$\operatorname{AUTHOR}(S):$

Hayakawa, $\mathrm{H}$

\section{CITATION:}

Hayakawa, H. Slow viscous flows in micropolar fluids. Physical Review E 2000, 61(5): 5477-5492

ISSUE DATE:

2000-05

URL:

http://hdl.handle.net/2433/50476

RIGHT:

Copyright 2000 American Physical Society 


\title{
Slow viscous flows in micropolar fluids
}

\author{
Hisao Hayakawa \\ Graduate School of Human and Environmental Studies, Kyoto University, Sakyo-ku, Kyoto 606-8501, Japan
}

(Received 13 September 1999; revised manuscript received 7 January 2000)

\begin{abstract}
A systematic calculation of micropolar fluid flows around a sphere and a cylinder is presented. The explicit velocity fields and the drag forces exerted by the fluid flow in both two and three dimensions are obtained. The solution of a steady micropolar fluid flow inside the cylinder is also obtained and is identical to the form observed in an experiment on granular vibrating beds.
\end{abstract}

PACS number(s): 45.70.Mg, 47.15.Gf, 83.50.Jf

\section{INTRODUCTION}

Micropolar fluids are fluids with microstructures. They belong to a class of fluids with a nonsymmetric stress tensor. Micropolar fluids consist of rigid, randomly oriented (or spherical) particles with their own spins and microrotations, suspended in a viscous medium. The concept of microrotation was proposed by Cosserat and Cosserat in the theory of elasticity [1]. Condiff and Dahler [2] and Eringen [3] applied the concept to describe fluids with microstructures in the middle of the 1960s. Recently, a comprehensive textbook on micropolar fluids has been published [4].

Physical examples of micropolar fluids can be seen in ferrofluids [5], blood flows [6,7], bubbly liquids [8], liquid crystals [9], and so on, all of them containing intrinsic polarities. Thus, micropolar fluid mechanics is not a useless generalization of the Navier-Stokes model, but is a physically relevant model that has many applications.

The most interesting application of micropolar fluid mechanics is to describe granular flows [10-14]. In fact, granular flow is one of the flows that have microstructure and rotation of particles. Thus, Kanatani [15] has formulated a micropolar fluid model for granular flows. Kano et al. [16] have confirmed the quantitative validity of the micropolar fluid model in a chute flow of granular particles by comparison of their simulation of micropolar fluids with their experiments. It is worthwhile to indicate that the velocity profile of the chute flow (vertical component to the slope) obtained from the micropolar fluid model [16] is far from the parabolic curve expected from the conventional Navier-Stokes flow, but is similar to a linear function when the slope is not large. For larger slopes, the profile becomes concave (i.e. the power index is smaller than 1) with surface slips. Although we do not know whether the micropolar fluid model is applicable in other situations of granular flow, it is worthwhile to investigate fundamental properties of micropolar fluids from the viewpoint of granular physics.

In this paper, we focus on slow viscous flows of micropolar fluids. The main purpose of this study is to clarify the mathematical structure of the creep flow in a Newtonian micropolar fluid model. This motivation is independent of our interest in granular flows. The main reason why we adopt the Newtonian model is its simplicity and its generality. We also look for the possibility of applying the Newtonian micropolar fluid model to granular flows as a phenomenological description.
If we admit the relevancy of micropolar fluid mechanics, it is obvious that the flow around a cylinder or a sphere and its drag force play fundamental roles. Thus, we will calculate the fluid flows under such circumstances. We will also discuss a steady flow inside a container. The result inside a cylinder is identical to that observed in an experiment on vibrating beds [17]. We will comment on the steady flow inside a rectangular container, which also gives identical results to those in experiments and simulations $[17,18]$.

There are some relevant previous investigations on creep flows in micropolar fluid mechanics [4]. In particular, Ramkissoon [19] has obtained the solution of a micropolar fluid flow around a sphere and the drag force exerted on the sphere. Later, Power and Ramkissoon [20] presented a fundamental solution, i.e., the Green function, etc., the Stokesian micropolar flow. It seems, however, that Ramkissoon's calculation [19] contains minor mistakes. Thus, we may need a revised calculation. Although Buchukuri and Chichinadze [21] obtained the fundamental solution and predicted the fluid flow around a cylinder as an integral form, they could not present the explicit velocity field and the drag force. Here, we will give explicit expressions based on the algorithm by Kaplun and Lagerstrom [22-26].

The organization of this paper is as follows. In the next section, we will briefly review studies of granular hydrodynamics. This section consists of three parts. The first part is devoted to introduction of the difficulties of the conventional Chapman-Enskog approach to deriving hydrodynamic equations from kinetic theory. In the second part we will explain the outline of Kanatani's formulation of granular hydrodynamics based on micropolar fluid mechanics [15]. In the last part of this section, we will discuss whether Newtonian micropolar fluid mechanics can be used in granular flows. In Sec. III, we will explain the general framework for a steady viscous flow around a sphere or a cylinder. In Sec. IV, we will show the correct calculation of the Stokes flow around a sphere and the drag force. We correct the result obtained by Ramkissoon [19]. In Sec. V, we will obtain an explicit solution of the micropolar fluid model around a cylinder by the method of Kaplun and Lagerstrom [22-26]. We calculate the drag force exerted on the cylinder. In Sec. VI, we will calculate the axial symmetric flow inside a cylinder and a rectangular container. We demonstrate that flows observed in experiments and simulations are similar to the solutions of the micropolar fluid model. In Sec. VII, we discuss the relevancy of our calculation in granular physics and how to 
improve it. We summarize general features of micropolar fluid mechanics, and conclude our results.

\section{REVIEW OF GRANULAR HYDRODYNAMICS}

In this section, we present a brief review of granular hydrodynamics. This review may clarify our motivation to apply micropolar fluid mechanics to granular flows. If readers are not interested in its application to granular flows but are interested in micropolar fluid mechanics itself, they can ignore this section.

\section{A. Conventional approaches and their difficulties}

It is natural to adopt hydrodynamic approaches to characterize granular flows because flows should be described by a kind of fluid mechanics based on Euler's description. However, it is obvious that any continuous description has limitations, because granular materials consist of visible grains. Eventually, any fluid model cannot be a microscopic model but a phenomenology. However, if we look for models that have a microscopic basis, we have to begin with molecular dynamics or the distinct element method (DEM) [27]. (Even the DEM contains many phenomenological assumptions which have not been justified from the theory of elasticity [28].) As is well known, simulations of the DEM are not always helpful in understanding the mechanism of granular flow. Thus, what we need is a good phenomenology that can apply to many phenomena.

One popular approach to describing granular flows is to adopt hydrodynamic equations derived from a kinetic equation like the Boltzmann equation based on the ChapmanEnskog scheme. This approach has been successful in describing molecular gas kinetics and in deriving the NavierStokes equation. However, this approach is not free from phenomenology when the density of particles is high. Since granular systems cannot be uniformly dilute [29], this defect is serious for granular hydrodynamics. In particular, when we are interested in dense flows under the effects of gravity, this approach cannot be justified from microscopic physics. Although the Enskog equation is sometimes used as a microscopic starting equation instead of the Boltzmann equation, its derivation cannot be justified even in systems without dissipation [30].

Hydrodynamics based on gas kinetics may be applicable to rapid granular flows [31] where the system is kept in relatively low density and does not include any stagnant region. Savage and Jeffrey [32] calculated the stress tensor from a modified Enskog equation, taking into account effects of inelastic collisions. The result can be non-Newtonian when the shear is strong and Newtonian when the shear is weak. Their method is applicable to dry debris flows [33]. Their analysis based on the kinetic equation is summarized in Refs. [3436] and some of their results are supported by experiment [37]. Haff's phenomenological granular hydrodynamics [38] is consistent with that obtained from the kinetic approach. Thus, granular hydrodynamics was accepted as being supported by microscopic physics in the 1980s.

In the 1990s we have recognized that some assumptions used in granular hydrodynamics are violated. Systems cannot be isotropic and homogeneous. Even if we start from dilute and homogeneous initial conditions, granular gases without gravity form stringlike clusters in which the density is very high. Thus, it has become the consensus that hydrodynamics cannot be used, at least in one-dimensional systems [39-41]. Similarly, Brey et al. [42] have shown that the velocity distribution function obeys a power law, and hydrodynamic equations break down when the restitution constant exceeds a critical value.

On the other hand, there are some applications of granular hydrodynamics as phenomenology. Bourzutschky and Miller [43] applied hydrodynamics with the slip boundary condition to granular convection in vibrating beds. Hayakawa et al. [44] also proposed a hydrodynamic model of granular convection and discussed the mechanism of appearance of convection rolls. Hayakawa and Hong [45] compared the previous model [44] with a model with a relaxation term as a body force, similar to a model of traffic flows (the traffic model). They found that the traffic model is better than the previous one in capturing the characteristics of granular convective flow. Details of the discussion about the traffic model can be seen in Ref. [46]. However, the traffic model is a curious model which violates physical common sense. Let us briefly explain how the traffic model is different from other hydrodynamic models. The model consists of coupled equations for the density field $\rho$ and the velocity field $\mathbf{v}$ supplemented by its average $\overline{\mathbf{v}}$ as

$$
\begin{gathered}
\frac{\partial \rho}{\partial t}=-\boldsymbol{\nabla} \cdot(\rho \mathbf{v}), \\
\frac{\partial \mathbf{v}}{\partial t}+\mathbf{v} \cdot \boldsymbol{\nabla} \mathbf{v}=-\zeta(\rho)(\mathbf{v}-\overline{\mathbf{v}})-\boldsymbol{\nabla} \cdot T,
\end{gathered}
$$

where $T$ is the stress tensor (assumed to be Newtonian in this analysis). A friction term proportional to $\zeta$ is not allowed in the usual one-phase hydrodynamics, because any short range interactions among particles reduce to the stress tensor $T$ in the continuum limit. However, it is interesting that Knight $e t$ al. [17] also suggested that their experimental results can be explained if a friction term as in Eq. (1) exists (and the pressure is negligible).

The onset of convective flow in vibrating beds, as well as the definition of viscosity in such circumstances are discussed in Ref. [47]. There is a report that fluid motion in vibrating beds is analogous to Fermi liquid theory [48]. Most hydrodynamic models to describe granular flows are assumed to be Newtonian models, however. The main reason why a Newtonian model is adopted is its simplicity for analysis. On the other hand, granular flow is obviously nonNewtonian in actual situations. We also note that in many of the models effects of dissipation appear only in the equation for energy balance.

\section{B. The outline of Kanatani's formulation}

As mentioned in Sec. II A, the hydrodynamic approaches based on an analogy with gas kinetics cannot be widely used. Thus, we had better seek another hydrodynamic approach which is more realistic in actual situations. In fact, appropriate hydrodynamic models have an industrial application, because particle simulations such as the DEM can treat only 1000000 particles, which is not a sufficiently large number 
to design actual plants. Note that the scaling of particle simulations is not so clear at present that we can extrapolate the results of simulations.

In this case we need to choose appropriate constitutive relations for the stress tensor. One successful one is by Goodman and Cowin [49], which obtains good agreement of the theoretical result for granular flow profile in a pipe with experiment [50]. We cannot generalize this success to other situations, however, because the model is too complicated.

Kanatani's formulation of micropolar fluid mechanics gives a general framework for granular hydrodynamics [15]. Kanatani's calculation of the pipe flow recovers the result by Goodman and Cowin [49]. Unfortunately, his paper is not well known because it is published in an engineering journal written in Japanese, but it is an important paper which includes careful considerations of physical processes.

His model consisting of particles with radius $a_{0}$ is assumed to obey the equations of continuity as

$$
\begin{gathered}
\frac{D \rho}{D t}=-\rho \boldsymbol{\nabla} \cdot \mathbf{v}, \\
\rho \frac{D \mathbf{v}}{D t}=\nabla \cdot T-\rho \mathbf{g}, \\
\frac{2}{5} \rho a_{0}^{2} \frac{D \boldsymbol{\omega}}{D t}=\nabla \cdot \mu_{T}+2 \widetilde{T} .
\end{gathered}
$$

Here $\mathbf{v}$ and $\boldsymbol{\omega}$ are the velocity and the microrotation, respectively. $D / D t=\partial_{t}+\mathbf{v} \cdot \boldsymbol{\nabla}$ is Lagrange's derivative, $\rho$ is the density, $T$ is the stress tensor, $\widetilde{T}$ is the asymmetric part of the stress tensor coming from consideration of the microrotation, $\mathbf{g}$ is the gravitational acceleration, and $\mu_{T}$ is the coupled stress tensor. $\frac{2}{5} \rho a_{0}^{2}$ in Eq. (4) represents the density of momentum inertia. This set of equations (2)-(4) is generally valid for any micropolar fluid.
Kanatani determined the stress tensor and the coupled stress tensor from physical considerations of microscopic granular motion. Kanatani's constitutive equation for the offdiagonal part of the stress tensor is summarized as

$$
\begin{aligned}
T_{j i}= & -p \delta_{j i}+C(\rho) \boldsymbol{\omega}_{a} \\
& \times\left[\frac{3}{10}\left(\frac{\partial v_{i}}{\partial x_{j}}+\frac{\partial v_{j}}{\partial x_{i}}-\frac{1}{3} \delta_{j i} \frac{\partial v_{k}}{\partial x_{k}}\right)+\frac{1}{2}\left(\frac{\partial v_{i}}{\partial x_{j}}-\frac{\partial v_{j}}{\partial x_{i}}-\omega_{j i}\right)\right]
\end{aligned}
$$

for developed flows and

$$
\begin{aligned}
T_{j i}= & -p \delta_{j i}+C_{K} \frac{p}{\boldsymbol{\omega}_{a}}\left[\frac{3}{10}\left(\frac{\partial v_{i}}{\partial x_{j}}+\frac{\partial v_{j}}{\partial x_{i}}-\frac{1}{3} \delta_{j i} \frac{\partial v_{k}}{\partial x_{k}}\right)\right. \\
& \left.+\frac{1}{2}\left(\frac{\partial v_{i}}{\partial x_{j}}-\frac{\partial v_{j}}{\partial x_{i}}-\omega_{j i}\right)\right]
\end{aligned}
$$

for slow flows. Here $\omega_{j i}=\partial v_{i} / \partial x_{j}-\partial v_{j} / \partial x_{i}, \quad C_{K}$ $=4 \sqrt{6 \pi} \mu a_{0}^{3} \rho /(3 m)$, and

$$
C(\rho)=8 \sqrt{6 \pi} T_{r} \mu d_{c}^{2} a_{0}^{3} \rho_{0} \rho^{2} /\left[15 m\left(\rho_{0}-\rho\right)\right],
$$

where $p, m, T_{r}, \rho_{0}$, and $\mu$ are, respectively, the pressure, the mass of a particle, the ratio of translational energy to rotational energy, the density for close packing, and Coulomb's friction constant. Kano et al. [16] replace $C(\rho)$ by

$$
C(\rho)=\frac{\sqrt{6} \mu e T_{r} d^{3} \rho}{30\left(d_{c}-d\right)}+\frac{\sqrt{10} T_{r} \sqrt{T_{r}} d^{3} \rho e(1-e)^{2}}{200\left(d_{c}-d\right)(1+e) \sin \varphi},
$$

where $d$ is the diameter, $d_{c}$ is the mean free path, and $\varphi$ and $e$ are the averaged scattering angle and the restitution constant, respectively. $\boldsymbol{\omega}_{a}$ contains the characteristics of particle dynamics whose explicit form is given by

$$
\boldsymbol{\omega}_{a}=\sqrt{\frac{3}{10} E_{j i} E_{j i}+\frac{1}{2} R_{j i} R_{j i}+\frac{d^{2}}{40}\left(\Omega_{k k j} \Omega_{l l j}+\Omega_{k j i} \Omega_{k j i}+\Omega_{k j i} \Omega_{k i j}\right)}
$$

with $E_{j i}=D_{(j i)}-\frac{1}{3} \delta_{j i} D_{k k}, D_{j i}=\partial v_{i} / \partial x_{j}, R_{j i}=\omega_{j i}=D_{[j i]}$, $\Omega_{k j i}=\partial \omega_{j i} / \partial x_{k}$, where $D_{(j i)}$ is the symmetric part of $D_{j i}$ and $D_{[j i]}$ is its asymmetric part.

The stress tensor in Eq. (5) is not far from a Newtonian one. In fact, if we assume that $\boldsymbol{\omega}_{a}$ is a constant, the model is reduced to a Newtonian one, although $\boldsymbol{\omega}$ cannot be a constant in general situations. We can show that Kanatani's model contains some improved characteristics as a hydrodynamic model for granular flows when we compare it with conventional ones coming from gas kinetics. The effect of dissipation appears in the equation of momentum balance, because the effective viscosity is proportional to Coulomb's friction constant. We also note that the radius of particles $a_{0}$ remains in the hydrodynamics. The model is reduced to the Navier-Stokes equation in the limit of $a_{0} \rightarrow 0$.
As mentioned in the Introduction, Kanatani's micropolar model gives a quantitatively accurate result for chute flows with minor modifications [16]. To reproduce the experimental results, the theory should include some of the characteristics of granular chute flows that are different from those of the Navier-Stokes equation. For example, there is a slip at the boundary between granular particles and containers [51]. There is the angle of repose for granular materials, and yield stress exists for the start of the slip motion. Kanatani's theory does include some of them. In fact, it includes the angle of repose $\theta_{*}$ as a parameter where $\tan \theta_{*}$ is proportional to $\mu$. It can describe the effective slip because of the particles' rotation. (That is, particles in the bottom layer can move with finite velocity under the nonslip boundary condition.) We also note that micropolar fluid mechanics including Kanatani's theory contains a relaxation term in the equation for 
the microrotation (4) which reproduces physically reasonable results [as in Eq. (1) of the traffic model [46]].

\section{Possible application of the micropolar model to granular flows}

It is obvious that there are gaps between granular flows and the micropolar fluid model. The most serious objection is that granular materials have completly different static properties from any kind of fluids that do not have shear stress, while continuous models of the granular materials have shear stress. The saturation of the pressure is known as Janssen's law [52], which is derived from a continuous model with a suitable constitutive relation. The concept of the pressure might thus be ill-posed even in slow granular flows $[53,54]$. As a result, slow fluid properties are expected to be considerably different from what we expect from Newtonian flows [55]. In fact, granular flows sometimes coexist with the stagnant regions. Since statics is out of our scope at least in this paper, we cannot characterize such a coexistence of statics and dynamics in granular materials with the micropolar fluid model.

However, the above objection may not be fatal, for the following reasons. (i) Continuous approaches for statics are accepted as standard [56-59]. (ii) If the effect of the sidewall is negligible, one of the directions of the principal stress is vertical for active states (the static mode) of continuous granules. Along this direction there is no shear stress (i.e., the tensor is diagonalized) and the static pressure is identical to that in a stationary fluid [52]. Thus, if we discuss the dynamic properties of granular flows far from the boundary, we may expect that fluid models can be used to describe the characteristics of granular flows. (iii) After the flow is generated in such a situation, it can be maintained easily. Thus, the static properties are suppressed in these cases. (iv) The experiment and simulation by Kano et al. [16] strongly suggest the relevancy of the micropolar fluid model in nonstagnant granular flows.

The second serious objection is that the granular flow is not Newtonian. In fact, Kanatani's micropolar granular fluid model $[15,16]$ is a strongly nonlinear and non-Newtonian fluid. However, (i) our main aim is to study the fundamental properties of micropolar fluid mechanics, which is needed to check the validity of micropolar fluid models for granular flow. For this purpose Newtonian models are appropriate because they can be investigated by the analytic method. (ii) The flow of granular materials can be smooth when the shear rate exceeds the yield value, and flows are metastable when the shear rate is a little lower than the yield. This kind of smooth flow may be approximated by Newtonian flow. (iii) The profile of granular flow in a pipe is similar to the solution of the Newtonian micropolar fluid model, namely, the solutions of the Newtonian micropolar fluid model are far from those of the Navier-Stokes equation. For example, the exact solution of Poiseuille flow between parallel plates for a Newtonian micropolar fluid under the nonslip boundary condition $[4,60]$ can describe plug flow $[49,50,61,62]$. If the boundary condition is assumed to be nonslip, the profile continuously changes from parabolic to plug flow depending on the coefficient of restitution [62]. The profile of twodimensional (2D) Poiseuille flow in the range $y=[-1,1]$ under the nonslip boundary condition is given by [4]

$$
v_{x}(y)=1-y^{2}-2 \sqrt{\frac{2 \mu_{r}}{\mu_{B}}} \frac{\cosh (k)-\cosh (k y)}{\sinh (k)},
$$

where the flow direction is $x$ and $k=\sqrt{\mu_{r} \mu_{B} / 8}$ with the dimensionless viscosities of micro-rotation $\mu_{r}$ and $\mu_{B}$. Note that we need translation of the parameters from Eq. (3.1.9) of Ref. [4] into Eq. (9), because we adopt the dimensionless parameters introduced in this paper. Equation (9) is similar to previous reports on granular pipe flows [49], though the density is not uniform in actual granular flows. It is interesting that the simulation by Peng and Ohta [62] is similar to Eq. (9). They adopt the lattice gas cellular automata method which does not introduce any explicit rotation of particles. Thus, the microrotation may not have to be identified with the rotation of particles. (iv) Many models under steady flows may reduce to a linearized Newtonian model in the viscous limit, which is also physically relevant as creep flow in actual situations. (v) The micropolar fluid model for granular flows by Kanatani $[15,16]$ can actually reduce to the Newtonian model when we assume a constant $\boldsymbol{\omega}_{a}$ ( the preaveraging approximation). Thus, from the great success of Kanatani's micropolar fluid mechanics in chute flows, it is natural to investigate fundamental properties of its simplified model. (vi) Most granular hydrodynamic models are assumed to be Newtonian. In other words, we expect that there are some common features of granular flows which may not disappear under the assumption of Newtonian flow.

\section{GENERAL FRAMEWORK}

In this section, we present the general framework for the calculation of slow micropolar fluid flows. The contents are independent of the granular hydrodynamics discussed in the previous section.

Let us restrict our interest to steady viscous flow around/ inside a sphere or a cylinder, i.e., $\partial / \partial t=0$ in Eqs. (2)-(4). For simplicity, let us use dimensionless quantities for later discussion, which are normalized by the velocity far from the sphere or the cylinder (in cases of flow past a sphere or a cylinder), and the radius of the sphere or the cylinder.

Thus, we start from the following set of equations. Slow flows satisfy the incompressible condition because the advection term $\mathbf{v} \cdot \boldsymbol{\nabla} \mathbf{v}$ is negligible. The incompressible condition is given by

$$
\operatorname{div} \mathbf{v}=0,
$$

where $\mathbf{v}$ is the velocity field. The equation for momentum conservation is [4]

$$
R \mathbf{v} \cdot \boldsymbol{\nabla} \mathbf{v}=-\nabla p+\Delta \mathbf{v}+\mu_{r} \operatorname{rot} \boldsymbol{\omega}
$$

where $\Delta$ is the Laplacian, $R$ is the effective Reynolds number, $p$ is the pressure, $\boldsymbol{\omega}$ is the microrotation, and $\mu_{r}$ is the dimensionless viscosity of the microrotation field which is assumed to be less than 2. Note that the Reynolds number $R$ and $\mu_{r}$ are represented by quantities with physical units as $R=\rho U a /\left(\eta+\eta_{r}\right)$ and $\mu_{r}=2 \eta_{r} /\left(\eta+\eta_{r}\right)$, where $\rho, U, a$, $\eta$, and $\eta_{r}$ are the density, the magnitude of the characteristic flow, e.g., the flow far from a sphere/cylinder, the radius of 
the sphere/cylinder, the conventional viscosity, and the viscosity for microrotation, respectively. The flow of microrotation is governed by

$$
\frac{R I}{\mu_{r}} \mathbf{v} \cdot \boldsymbol{\nabla} \boldsymbol{\omega}=\operatorname{rot} \mathbf{v}-2 \boldsymbol{\omega}+\mu_{A} \nabla \operatorname{div} \boldsymbol{\omega}+\mu_{B} \Delta \boldsymbol{\omega},
$$

where $I$ is the dimensionless microinertia coefficient [4]. In general, $\operatorname{div} \boldsymbol{\omega}$ is not equal to zero but it is easy to show that

$$
\operatorname{div} \boldsymbol{\omega}=0
$$

for axial symmetric flows. Later we will discuss axially symmetric situations and assume Eq. (13). Thus, we regard the microrotation field as incompressible, where the term proportional to $\mu_{A}$ in Eq. (12) is zero in later discussion. Let us remark on the micropolar fluid model. It is obvious that the model is reduced to the Navier-Stokes equation for $\mu_{r} \rightarrow 0$.

We assume the boundary condition outside the sphere/cylinder as

$$
\mathbf{v}=\boldsymbol{\omega}=0 \quad \text { at } \quad r=1 ; \quad \mathbf{v}=\mathbf{e}_{x}, \quad \boldsymbol{\omega} \longrightarrow \frac{1}{2} \operatorname{rot} \mathbf{v} \quad \text { as } \quad r \rightarrow \infty,
$$

where $r$ is the distance from the center of the sphere or the cylinder whose radius is unity, and $\mathbf{e}_{x}$ is the unit vector along the $x$ axis. The above boundary conditions are not always valid in micropolar fluids. We assume the nonslip boundary condition on the boundary surface, which ensures continuity of flow in the entire region. Effective slip of particles is included as microrotation. The microrotation on the surface is assumed to be zero because the center of rotation cannot exist on the surface but exists at a position removed by the particle radius. The microrotation coincides with the rotation of the flow $\mathbf{v}$ if the place is for enough away from the sphere or cylinder.

On the other hand, inside the cylinder, we assume an axially symmetric flow $\mathbf{v}=\left(v_{r}(r, z), 0, v_{z}(r, z)\right)$. The boundary condition is assumed to be

$$
v_{r}=\frac{\partial v_{z}}{\partial r}=0 \quad \text { at } \quad r=0, \quad v_{r}=0 \quad \text { at } \quad r=1
$$

In addition to Eq. (15) we have a conservation law,

$$
\int_{0}^{1} d r r v_{z}(r, z)=0
$$

For two-dimensional cases (flows inside a box where the horizontal coordinate $x$ satisfies $|x| \leqslant 1$ and the vertical coordinate is $x$ ), Eqs. (15) and (16) are replaced by

$$
v_{z}=\frac{\partial v_{z}}{\partial x}=0 \quad \text { at } \quad x=0, \quad v_{x}=0 \quad \text { at } \quad x= \pm 1
$$

and

$$
\int_{-1}^{1} d x v_{z}(x, z)=0
$$

Note that we do not impose $v_{z}=0$ at $r=1$ for $3 \mathrm{D}$ or at $x=$ \pm 1 for $2 \mathrm{D}$, because this condition produces an overcomplete problem. Thus the problem inside a cylinder or a rectangular container is rather more specialized than flow around a cylinder/sphere. There are some unclear points in setting up the problem for flow inside a cylinder.

What we will do is to carry out a systematic calculation of the flow field around a sphere or cylinder. For this purpose, we adopt the matched asymptotic method developed by Kaplun and Lagerstrom [22-26] for the 2D problem. There are several advantages of this scheme. First of all, this method can discuss the systematic expansion of the Reynolds number. Second, this method can simplify complicated calculations. For example, Buchukuri and Chichinadze [21] could not obtain an explicit solution of the Oseen approximation of 2D fluid flow. However, as will be shown, even in 2D we do not have to solve the Oseen problem for the micropolar fluid model explicitly but need the Oseen solution for standard fluid flow.

Let us explain the second advantage more explicitly. It is well known that the Stokes approximation $\left[R=0\right.$ and $\mu_{r}$ $=0$ in Eq. (11)] is invalid far from the cylinder, and a naive perturbative calculation from the Stokes approximation encounters a secular term for the flow around a sphere. Therefore, in micropolar fluids, we need careful treatments to calculate the flow around a cylinder/sphere. To remove such difficulties we introduce an appropriate contracted coordinate as

$$
\tilde{x}=R x, \quad \tilde{y}=R y .
$$

Let us introduce scaled variables

$$
\mathbf{v}=\mathbf{e}_{x}+\alpha(R) \mathbf{u}(\tilde{\mathbf{r}}), \quad \boldsymbol{\omega}=\alpha(R) R \tilde{\omega} \mathbf{e}_{\perp}, \quad p=R \alpha(R) \tilde{p},
$$

where $\mathbf{e}_{\perp}$ is the unit vector vertical to the flow direction. The function $\alpha(R)$ will be determined by the matching. Thus, e.g., in the $2 \mathrm{D}$ case Eqs. (11) and (12) are reduced to

$$
\begin{gathered}
\frac{\partial \mathbf{u}}{\partial \tilde{x}}=-\tilde{\nabla} \tilde{p}+\tilde{\Delta} \mathbf{u}+\mu_{r} \tilde{\operatorname{rot}}\left(\tilde{\omega} \mathbf{e}_{z}\right), \\
\frac{R I}{\mu_{r}} \frac{\partial \tilde{\omega}}{\partial \tilde{x}}=\frac{\partial u_{y}}{\partial \tilde{x}}-\frac{\partial u_{x}}{\partial \tilde{y}}-2 \tilde{\omega}+\mu_{B} R^{2} \tilde{\Delta} \tilde{\omega},
\end{gathered}
$$

where $\mathbf{e}_{\perp}$ is reduced to $\mathbf{e}_{z}$ in 2D. Thus, in the limit of $R$ $\rightarrow 0$, we obtain the two relations

$$
\tilde{\omega}=\frac{1}{2}\left(\frac{\partial u_{y}}{\partial \tilde{x}}-\frac{\partial u_{x}}{\partial \tilde{y}}\right) ; \quad \tilde{\operatorname{rot}}\left(\tilde{\omega} \mathbf{e}_{z}\right)=-\frac{1}{2} \tilde{\Delta} \mathbf{u}
$$

The situation in $3 \mathrm{D}$ is almost the same as that in $2 \mathrm{D}$. The outer equations of the micropolar fluid are thus reduced to

$$
\frac{\partial \mathbf{u}}{\partial \tilde{x}}=-\tilde{\nabla} \tilde{p}+\left(1-\frac{\mu_{r}}{2}\right) \tilde{\Delta} \mathbf{u}+O(R)
$$

in both the 2D and 3D problems. The solution of Eq. (24) is regular even far from the cylinder. As a result, we do not have to solve the Oseen approximation of Eqs. (11) and (12), which cannot be represented by an explicit form [21]. Equa- 
tion (24) supports the validity of the boundary condition $\boldsymbol{\omega}$ $\rightarrow \operatorname{rot} \mathbf{v} / 2$ as $r \rightarrow \infty$ in Eq. (14).

Since we know the solution of Eq. (24) for 2D and 3D, what we need to solve is the Stokes approximation of Eqs. (11) and (12) in an axially symmetric flow $\boldsymbol{\omega}=\omega \mathbf{e}_{\perp}$ as

$$
-\nabla p+\Delta \mathbf{v}+\mu_{r} \operatorname{rot}\left(\omega \mathbf{e}_{\perp}\right)=0
$$

and

$$
(\operatorname{rot} \mathbf{v})_{\perp}-2 \omega+\mu_{B} \Delta \omega=0
$$

near the cylinder or the sphere. The solutions of Eq. (24) and Eqs. (25),(26) will be connected with the aid of the matching asymptotic technique.

\section{FLOW PAST A SPHERE}

In this section let us look for a solution to describe a three-dimensional steady flow past a sphere. The vectors $\mathbf{v}$ and $\boldsymbol{\omega}$ can be represented by their elements in polar coordinates as $\mathbf{v}=\left(v_{r}, v_{\theta}, v_{\varphi}\right)$ and $\boldsymbol{\omega}=\left(\omega_{r}, \omega_{\theta}, \omega_{\varphi}\right)$, where the polar axis is identical to the $x$ axis and its origin is the center of the sphere. Here $\mathbf{e}_{\perp}$ in the previous section is replaced by $\mathbf{e}_{\varphi}$. Under the assumption of axially symmetric flow with $v_{\varphi}$ $=0$, there is a stream function which is related to the velocity field

$$
v_{r}=\frac{1}{r^{2} \sin \theta} \frac{\partial \Psi}{\partial \theta} ; \quad v_{\theta}=-\frac{1}{r \sin \theta} \frac{\partial \Psi}{\partial r}
$$

in three-dimensional systems. On the other hand, e.g., $\omega_{r}$ and $\omega_{\theta}$ are constants which are independent of $\mathbf{v}$. So we assume $\omega_{r}=\omega_{\theta}=0$ and

$$
\omega_{\varphi}=\frac{\Phi}{r \sin \theta}
$$

in $3 \mathrm{D}$.

In $3 \mathrm{D}$ polar coordinates, there are the useful relations

$$
(\operatorname{rot} \mathbf{v})_{\varphi}=-\frac{1}{r \sin \theta} D^{2} \Psi
$$

and

$$
\Delta(\operatorname{rot} \mathbf{v})_{\varphi}=-\frac{1}{r \sin \theta} D^{4} \Psi
$$

where the differential operator $D^{2}$ is defined by

$$
D^{2}=\left[\frac{\partial^{2}}{\partial r^{2}}+\frac{\sin \theta}{r^{2}} \frac{\partial}{\partial \theta}\left(\frac{1}{\sin \theta} \frac{\partial}{\partial \theta}\right)\right] .
$$

Thus, it is convenient to represent the flow by the stream function $\Psi$.

\section{A. Stokes flow}

Let us consider the solution of the Stokes equations (25) and (26). The method of calculation itself is essentially the same as that by Ramkissoon [19]. In such a case from the divergence of Eq. (25) we obtain

$$
\Delta p=0 .
$$

That is, the pressure is a harmonic function. On the other hand, the rotation of Eq. (25) is

$$
\Delta \operatorname{rot} \mathbf{v}+\mu_{r} \operatorname{rot} \operatorname{rot} \omega=0 .
$$

Substituting Eqs. (28) and (30) into Eq. (33) with the aid of an elementary formula of vector analysis, we obtain

$$
D^{4} \Psi+\mu_{r} D^{2} \Phi=0 .
$$

On the other hand, substituting Eq. (29) into Eq. (26) we obtain

$$
D^{2} \Psi+\left(2-\mu_{B} D^{2}\right) \Phi=0 .
$$

From Eqs. (30) and (34), Eq. (35) can be rewritten as

$$
D^{2}\left(D^{2}-\xi^{-2}\right) \Phi=0,
$$

where

$$
\xi=\sqrt{\frac{\mu_{B}}{2-\mu_{r}}} .
$$

Note that Eq. (2.5) in Ramkissoon's paper [19], which can be translated into

$$
\Phi=\frac{1}{2}\left(D^{2} \Psi+\frac{\mu_{B}}{\mu_{r}} D^{4} \Psi\right),
$$

is not correct, but the correct correspondence from Eqs. (34) and (35) is given by

$$
\Phi=-\frac{1}{2}\left(D^{2} \Psi+\frac{\mu_{B}}{\mu_{r}} D^{4} \Psi\right) .
$$

As a result, Ramissoon's calculation [19] leads to inaccurate results.

The boundary conditions (14) now reduce to

$$
\Psi(1, \theta)=0, \quad \frac{\partial \Psi}{\partial r}(1, \theta)=0, \quad \Phi(1, \theta)=0
$$

on the surface of the sphere, and the asymptotic conditions as $r \rightarrow \infty$ are

$$
\Psi \rightarrow \frac{1}{2} r^{2} \sin \theta ; \quad \Phi \rightarrow-\frac{1}{2} D^{2} \Psi,
$$

where we use Eqs. (28) and (29). Thus, the problem is reduced to a boundary value problem of Eqs. (34) and (36) under the boundary conditions (40) and (41).

Equations (34) and (36) are fourth order partial differential equations. A reduced variable $\phi=D^{2} \Phi$ satisfies

$$
\left(D^{2}-\xi^{-2}\right) \phi=0 .
$$

Assuming the separation of variables as $\phi=R_{\phi}(r) \Theta_{\phi}(\theta)$ we obtain

$$
r^{2}\left(\frac{R_{\phi}^{\prime \prime}}{R_{\phi}}-\xi^{-2}\right)=-\frac{\sin \theta}{\Theta_{\phi}} \frac{d}{d \theta}\left(\frac{1}{\sin \theta} \frac{d \Theta_{\phi}}{d \theta}\right)=\lambda_{\phi},
$$


where $\lambda_{\phi}$ is a separation constant. The equation for $\Theta_{\phi}$ is reduced to

$$
\left(1-z^{2}\right) \frac{d^{2} w}{d z^{2}}-2 z \frac{d w}{d z}+\left(\lambda_{\phi}-\frac{1}{1-z^{2}}\right) w=0
$$

where $z=\cos \theta$ and $\Theta_{\phi}=(\sin \theta) w(\cos \theta)$. Equation (44) is the equation for Legendre's bipolynomial $P_{l}^{1}(z)$. Thus we obtain

$$
\Theta_{\phi, l}(\theta)=(\sin \theta) P_{l}^{1}(\cos (\theta)) ; \quad \lambda_{\phi}=l(l+1)
$$

where $l$ is a positive integer. The radial equation in Eq. (43) obeys

$$
\frac{d^{2} R_{\phi, l}}{d r^{2}}-\left(\xi^{-2}+\frac{l(l+1)}{r^{2}}\right) R_{\phi, l}=0
$$

where we write the explicit dependence of $R_{\phi}$ on $l$ as $R_{\phi, l}$. This equation can be converted into the equation for a modified Bessel function. Taking into account the regularity of $r$ $\rightarrow \infty$, the solution $R_{\phi, l}$ must be proportional to the modified Bessel function $K_{l+1 / 2}(r / \xi)$. Thus, the solution of $\phi$ can be written as

$$
\phi(r, \theta)=\sum_{l=1}^{\infty} C_{l} \sqrt{\frac{r}{\xi}} K_{l+1 / 2}\left(\frac{r}{\xi}\right) \sin \theta P_{l}^{1}(\cos \theta) .
$$

Note that $K_{l+1 / 2}(r / \xi)$ can be represented by an elementary function because modified Bessel functions of fractional order are proportional to spherical modified Bessel functions.

Let us obtain $\Phi$ from the equation $D^{2} \Phi=\phi$. It is easy to show that $\Phi(r, \theta)=\sum_{l=1}^{\infty} R_{\Phi, l}(r) \Theta_{\Phi, l}(\theta)$ has the solution

$$
\Theta_{\Phi, l}=\Theta_{\phi, l}=\sin \theta P_{l}^{1}(\cos \theta) \text {. }
$$

Thus, the radial equation for $R_{\Phi, l}$ is reduced to

$$
\left(\frac{d^{2}}{d r^{2}}-\frac{l(l+1)}{r^{2}}\right) R_{\Phi, l}=C_{l} \sqrt{\frac{r}{\xi}} K_{l+1 / 2}\left(\frac{r}{\xi}\right) .
$$

The general solution of Eq. (49) is given by a linear combination of homogeneous solutions and a special solution of the inhomogeneous equation. The independent homogeneous solutions of Eq. (49) are $r^{l+1}$ and $r^{-l}$. From direct substitution, it is easy to show a special solution $R_{\Phi, l}^{N H S}$,

$$
R_{\Phi, l}^{N H S}=\xi^{2} C_{l} \sqrt{\frac{r}{\xi}} K_{l+1 / 2}\left(\frac{r}{\xi}\right) .
$$

Thus, $\Phi(r, \theta)$ can be represented by

$$
\begin{aligned}
\Phi(r, \theta)= & \sum_{l=1}^{\infty}\left[a_{\Phi, l} r^{l+1}+\frac{b_{\Phi, l}}{r^{l}}\right. \\
& \left.+C_{l} \xi^{2} \sqrt{\frac{r}{\xi}} K_{l+1 / 2}\left(\frac{r}{\xi}\right)\right] \sin \theta P_{l}^{1}(\cos \theta) .
\end{aligned}
$$

Similarly, from Eqs. (34) and (51) we obtain

$$
\Psi(r, \theta)=\sum_{l=1}^{\infty} R_{\Psi, l}(r) \sin \theta P_{l}^{1}(\cos \theta),
$$

where

$$
\begin{aligned}
R_{\Psi, l}(r)= & a_{\Psi, l} r^{l+1}+\frac{b_{\Psi, l}}{r^{l}}+c_{\Psi, l} r^{l+3}+d_{\Psi, l} r^{2-l} \\
& -\mu_{r} C_{l} \xi^{4} \sqrt{\frac{r}{\xi}} K_{l+1 / 2}\left(\frac{r}{\xi}\right)
\end{aligned}
$$

These are general results for outer solutions without explicit considerations of boundary conditions.

From the boundary condition (41) there are the following relations:

$$
a_{\Phi, l}=c_{\Psi, l}=0 \quad(\text { for all } l) ; \quad a_{\Psi, 1}=\frac{1}{2}, \quad a_{\Psi, l}=0 \quad(l \geqslant 2) .
$$

From the boundary conditions (40) and (41) we can determine $b_{\Psi, l}, d_{\Psi, l}, C_{l}$, and $b_{\Phi, l}$. However, for $l \geqslant 2$, we have vector equations

$$
A_{l} \mathbf{x}_{l}=\mathbf{0}, \quad l \geqslant 2,
$$

where $A_{l}$ is the matrix determined through the boundary conditions, and the transverse of $\mathbf{x}_{l}$ is ${ }^{T} \mathbf{x}_{l}$ $=\left(b_{\Psi, l}, d_{\Psi, l}, C_{l}, b_{\Phi, l}\right)$. Since Eq. (55) should hold for any parameters, $\operatorname{det} A_{l}$ cannot be zero in general. Thus we have

$$
b_{\Psi, l}=d_{\Psi, l}=C_{l}=b_{\Phi, l}=0
$$

for $l \geqslant 2$. Equations $(51)-(53)$ are now reduced to

$$
\Psi(r, \theta)=\left[\frac{1}{2} r^{2}+\frac{b}{r}+d r-\mu_{r} C \xi^{4} e^{-r / \xi}\left(1+\frac{\xi}{r}\right)\right] \sin ^{2} \theta,
$$

and

$$
\Phi(r, \theta)=\left[\frac{b_{\Phi}}{r}+C \xi^{2} e^{-r / \xi}\left(1+\frac{\xi}{r}\right)\right] \sin ^{2} \theta,
$$

where $b=b_{\Psi, 1}, d=d_{\Psi, 1}, \quad C=(\sqrt{\pi / 2}) C_{1}$, and $b_{\Phi}=b_{\Phi, 1}$. Since $D^{2} \Psi \rightarrow-(2 d / r) \sin ^{2} \theta$ as $r \rightarrow \infty$, the second relation of Eq. (41) leads to

$$
b_{\Phi}=d \text {. }
$$

(Note that Ramkissoon's result becomes $b_{\Phi}=-d$.) The other three boundary conditions in Eqs. (40) and (41) determine $b, d$, and $C$ as

$$
\begin{gathered}
b=-\frac{1}{2}+\frac{3(1+\xi)\left(1+\mu_{r} \xi^{2}\right)}{2\left[2(1+\xi)-\mu_{r} \xi\right]}, \\
d=-\frac{3(1+\xi)}{2\left[2(1+\xi)-\mu_{r} \xi\right]}, \\
C=\frac{3 e^{\xi^{-1}}}{2 \xi^{2}\left[2(1+\xi)-\mu_{r} \xi\right]} .
\end{gathered}
$$

Thus the problem has been solved.

From Eq. (27) the explicit profile of the velocity field is given by 


$$
\begin{gathered}
v_{r}=\left[1+\frac{2 b}{r^{3}}+\frac{2 d}{r}-2 \mu_{r} C \frac{\xi^{4}}{r^{2}}\left(1+\frac{\xi}{r}\right) e^{-r / \xi}\right] \cos \theta, \\
v_{\theta}=\left[-1+\frac{b}{r^{3}}-\frac{d}{r}-\mu_{r} C \frac{\xi^{3}}{r}\left(1+\frac{\xi}{r}+\frac{\xi^{2}}{r^{2}}\right) e^{-r / \xi}\right] \sin \theta,
\end{gathered}
$$

and $\omega_{\varphi}$ is

$$
\omega_{\varphi}=\left[\frac{d}{r^{2}}+C \frac{\xi^{2}}{r} e^{-r / \xi}\left(1+\frac{\xi}{r}\right)\right] \sin \theta
$$

The pressure is determined from Eq. (25). Each component obeys

$$
\begin{gathered}
\frac{\partial p}{\partial r}=\frac{1}{r^{2} \sin \theta} \frac{\partial}{\partial \theta}\left(D^{2} \Psi+\mu_{r} \Phi\right) ; \\
\frac{1}{r} \frac{\partial p}{\partial \theta}=-\frac{1}{r \sin \theta} \frac{\partial}{\partial r}\left(D^{2} \Psi+\mu_{r} \Phi\right) .
\end{gathered}
$$

It is easy to show that the terms proportional to the spherical modified Bessel function cancel with each other in Eq. (63) as expected from the properties of the harmonic function. The terms in proportion to $1 / r$ and $r^{2}$ in $\Psi$ disappear in Eq. (63) as in conventional cases. Thus, the pressure is determined by the Stokes pole of $\Psi$, which is proportional to $r$ as $\Psi_{s t}=d r \sin ^{2} \theta$ and to $1 / r$ in $\Phi$ as $\Phi_{s t}=(d / r) \sin ^{2} \theta$. From the relation $D^{2} \Psi_{s t}+\mu_{r} \Phi_{s t}=-\left[d\left(2-\mu_{r}\right) / r\right] \sin ^{2} \theta$ and Eq. (63), we obtain

$$
p=\frac{d\left(2-\mu_{r}\right)}{r^{2}} \cos \theta+p_{0},
$$

where $p_{0}$ is an unimportant constant.

The flow is similar to conventional Stokes flow, which is symmetrical about a plane normal to the external flow (Fig. 1 ). The most significant difference between the conventional Stokes flow and micropolar Stokes flow appears as a localized microrotation near the sphere (Fig. 2). The micropolar flow far from the sphere $r \gg \xi$ is not different from conventional flow.

\section{B. Drag force exerted on a sphere}

Let us calculate the force exerted on a sphere in a micropolar fluid flow. For this purpose we explicitly write the stress tensor $T_{i j}$ as

$$
\begin{aligned}
T_{i j}= & -p \delta_{i j}+\left(1-\frac{\mu_{r}}{2}\right)\left(\frac{\partial v_{j}}{\partial x_{i}}+\frac{\partial v_{i}}{\partial x_{j}}\right) \\
& +\frac{\mu_{r}}{2}\left(\frac{\partial v_{j}}{\partial x_{i}}-\frac{\partial v_{i}}{\partial x_{j}}\right)-\mu_{r} \epsilon_{m i j} \omega_{m}
\end{aligned}
$$

where $\epsilon_{m i j}$ is the alternative tensor of Levi-Cività. Now we decouple the stress tensor into two parts as

$$
T_{i j}=\sigma_{i j}+\tau_{i j} ; \quad \tau_{i j}=\mu_{r} \epsilon_{m i j}\left(\Omega_{m}-\omega_{m}\right),
$$

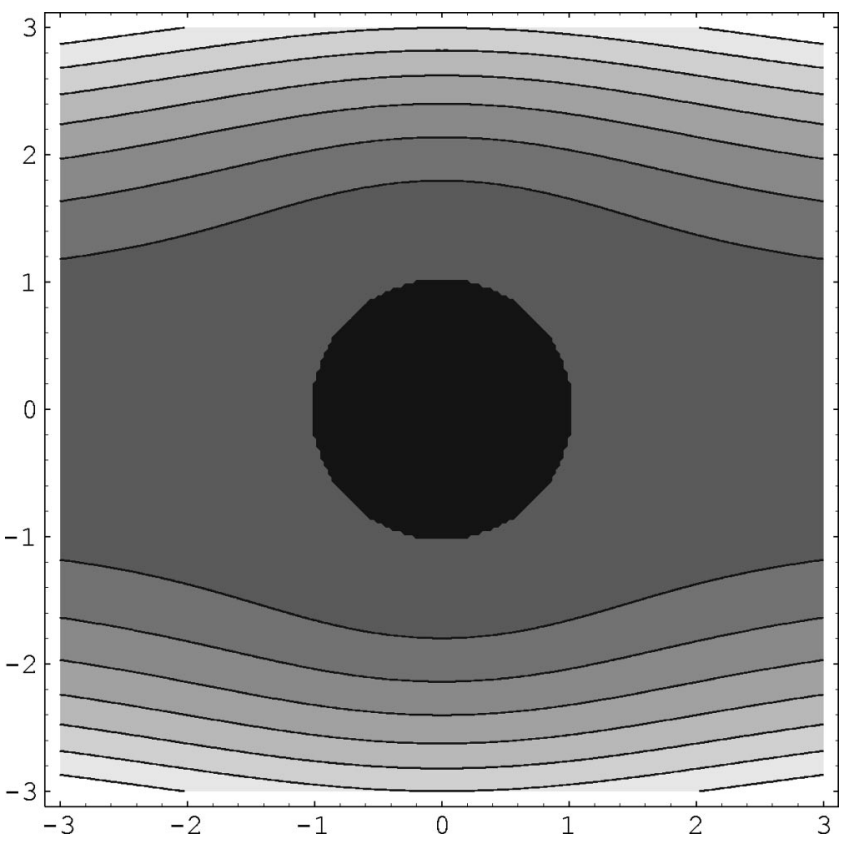

FIG. 1. The stream line of three-dimensional micropolar Stokes flow. The black circle represents the sphere. The flow direction is horizontal.

where $\Omega_{m}=(\operatorname{rot} \mathbf{v})_{m} / 2, \tau_{i j}$ is the asymmetric part of the stress tensor, and $\sigma_{i j}$ is the symmetric part.

Let us consider the contribution from $\tau_{i j}$ to the drag force. From considerations of symmetry, the drag force from this part is

$$
F_{\tau}=\left.\int_{S} \tau_{r x}\right|_{r=1} d S=\mu_{r} \int_{S}\left(-\frac{y}{r} \Omega_{z}+\frac{z}{r} \Omega_{y}\right) d S,
$$

where $d S$ represents the integration on the surface of the sphere. To derive the last expression in Eq. (67) we use Eq.

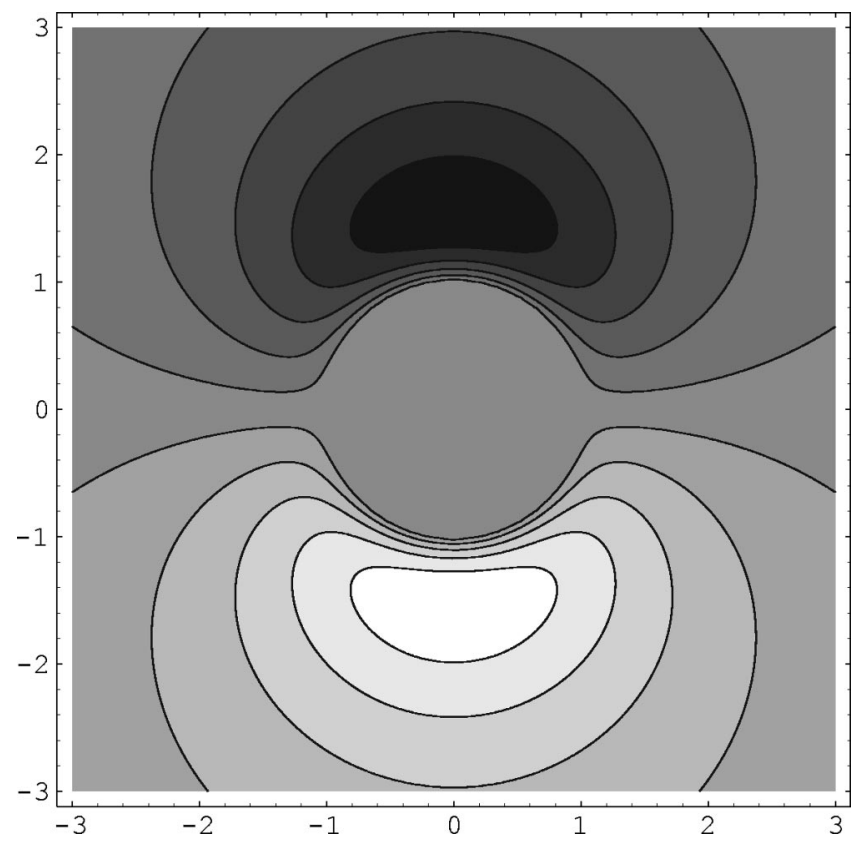

FIG. 2. Contour plot of the microrotation field. The situation is the same as that in Fig. 1. We have not plotted the sphere explicitly in this figure. 
(66) and $\boldsymbol{\omega}=0$ on the surface of the sphere. From $x / r$ $=\cos \theta, y / r=\sin \theta \cos \varphi$ and $z / r=\sin \theta \sin \varphi$, Eq. (67) is reduced to

$$
F_{\tau}=-\int_{0}^{2 \pi} d \varphi \cos \varphi \int_{0}^{\pi} d \theta \Omega_{\varphi}(1, \theta) \sin \theta=0 .
$$

Other contributions for the drag force correspond to those in the conventional Stokes problem. The contribution from the velocity field is given by

$$
\begin{aligned}
F_{v} & =-\left(1-\frac{\mu_{r}}{2}\right) \int_{S} d S \sin \theta \frac{\partial v_{\theta}}{\partial r}(1, \theta) \\
& =\frac{2 \pi\left(2-\mu_{r}\right)^{2}(1+\xi)}{2(1+\xi)-\mu_{r} \xi},
\end{aligned}
$$

where we use Eq. (61) and $\int_{0}^{\pi} d \theta \sin ^{3} \theta=4 / 3$. On the other hand, the contribution from the pressure term is

$$
F_{p}=-\int_{S} d S p \cos \theta=\frac{2 \pi\left(2-\mu_{r}\right)(1+\xi)}{2(1+\xi)-\mu_{r} \xi},
$$

where we use $\int_{0}^{\pi} d \theta \cos ^{2} \theta \sin \theta=2 / 3$ and Eqs. (64) and (60).

The total contribution $F_{v}+F_{p}+F_{\tau}$ is given by

$$
F_{t o t}=\frac{2 \pi(1+\xi)\left(2-\mu_{r}\right)\left(3-\mu_{r}\right)}{2(1+\xi)-\mu_{r} \xi} .
$$

Note that we have assumed $\mu<2$. The result can be converted into physical units. The drag force on the sphere (radius $a$ ) exerted by a micropolar fluid whose viscosities are $\eta$ and $\eta_{r}$ is given by

$$
F_{t o t}=\frac{2 \pi\left(\eta+\eta_{r}\right) a U(1+\xi)\left(2-\mu_{r}\right)\left(3-\mu_{r}\right)}{2(1+\xi)-\mu_{r} \xi},
$$

which is reduced to $6 \pi \eta a U$ as $\mu_{r}=2 \eta_{r} /\left(\eta+\eta_{r}\right) \rightarrow 0$ and $\xi \rightarrow 0$.

\section{Beyond the Stokes approximation}

In this subsection we briefly present the procedure to calculate the flow and the drag beyond the Stokes approximation. As mentioned in the Introduction, it is not possible to obtain an explicit representation of the flow field based on the Oseen approximation. Thus, it is natural to adopt the series expansion of the Reynolds number $R$. For this purpose we rewrite the full equations (11) and (12) for $\Psi$ and $\Phi$ as

$$
\begin{aligned}
& \frac{R}{r^{2} \sin \theta}\left(\frac{\partial \Psi}{\partial \theta} \frac{\partial}{\partial r}-\frac{\partial \Psi}{\partial r} \frac{\partial}{\partial \theta}+2 \cot \theta \frac{\partial \Psi}{\partial r}-\frac{2}{r} \frac{\partial \Psi}{\partial \theta}\right) D^{2} \Psi \\
& =D^{4} \Psi+\mu_{r} D^{2} \Phi
\end{aligned}
$$

and

$$
\begin{gathered}
\frac{R I}{\mu_{r} r^{2} \sin \theta}\left[\frac{1}{r} \frac{\partial \Psi}{\partial \theta}\left(1-r \frac{\partial}{\partial r}\right)+\frac{\partial \Psi}{\partial r}\left(\frac{\partial}{\partial \theta}-\cot \theta\right)\right] \Phi \\
=D^{2} \Psi+2 \Phi-\mu_{B} D^{2} \Phi .
\end{gathered}
$$

The solution can be obtained from

$$
\Psi=\Psi_{0}+R \Psi_{1}+\cdots, \quad \Phi=\Phi_{0}+R \Phi_{1}+\cdots,
$$

where $\Psi_{0}$ and $\Phi_{0}$ are the solutions in Stokes approximation in Eqs. (57) and (58). To obtain the explicit forms of $\Psi_{1}$ and $\Phi_{1}$ we need a long and tedious calculation. Thus, we do not give the explicit expressions.

However, we note that in the first order approximation the velocity does not behave properly at infinity. The nonexistence of a second approximation to the Stokes solution in conventional flow is known as Whitehead's paradox [25]. To remove this difficulty, we use the matched asymptotic method as introduced in Sec. II. That is, we use the outer solution determined from Eq. (24) far from the sphere, while we use the simple perturbative result as the inner solution. An unknown constant arising from the lack of a boundary condition far from the sphere is determined by matching the inner solution and the outer solution. Because Eq. (24) is solvable, it is straightforward to obtain the flow fields as series expansions of $R$. The explicit calculation will be discussed elsewhere.

\section{FLOW PAST A CYLINDER}

As is well known, for conventional viscous flow in $2 \mathrm{D}$, the solution based on the Stokes approximation does not satisfy the boundary condition far from the cylinder. This is known as Stokes' paradox and is more serious than Whitehead's paradox in 3D. Thus, we need to adopt the matched asymptotic method from the lowest order expansion of $R$.

In two-dimensional systems, the stream function is defined through

$$
v_{r}=\frac{1}{r} \frac{\partial \Psi}{\partial \theta} ; \quad v_{\theta}=-\frac{\partial \Psi}{\partial r}
$$

These choices automatically ensure Eq. (10). In 2D, the field variables are written as $\mathbf{v}=\left(v_{r}, v_{\theta}\right)$ and $\boldsymbol{\omega}=\omega_{z} \hat{\mathbf{z}}$ where $\hat{\mathbf{z}}$ is the unit vector vertical to the flow plane.

The procedure is as follows. In the next subsection we will give the explicit calculation of the Stokes approximation as an inner solution. In Sec. V B, we will obtain an outer solution and use the matched asymptotic method. In Sec. $\mathrm{VC}$, we will calculate the force exerted by the fluid flow.

\section{A. Stokes approximation as an inner solution}

At the first step, let us obtain the solution of Eqs. (25) and (26) based on the Stokes approximation. From Eqs. (76) and (33) we obtain an equation for $\omega_{z}$ and $\Psi$ :

$$
\Delta^{2} \Psi+\mu_{r} \Delta \omega_{z}=0 .
$$

On the other hand, from Eq. (26) we obtain

$$
\Delta \Psi+2 \omega_{z}-\mu_{B} \Delta \omega_{z}=0
$$

From the operation of the Laplacian on Eq. (78) with the help of Eq. (77) we obtain

$$
\Delta\left(\Delta-\xi^{-2}\right) \omega_{z}=0,
$$


where $\xi$ is given in Eq. (37). The problem will be solved under the boundary condition on the cylinder. Although we cannot adopt the boundary condition far from the cylinder, we know that the leading singularity comes from a logarithmic divergent term which will be regularized by matching with the outer solution. Thus, higher divergent terms which obey power laws will be omitted in discussion in this subsection. The boundary condition far from the cylinder is

$$
\omega_{z}=-\frac{1}{2} \Delta \Psi
$$

which is equivalent to $\omega_{z}=\operatorname{rot} \mathbf{v} / 2$.

Taking into account the boundary condition on the cylinder

$$
\Psi(1, \theta)=\frac{\partial \Psi}{\partial r}(1, \theta)=\omega_{z}(1, \theta)=0
$$

we obtain

$$
\Delta \omega_{z}(r, \theta)=\sum_{n=1}^{\infty} C_{n}^{(2)} K_{n}\left(\frac{r}{\xi}\right) \sin n \theta
$$

where $C_{n}^{(2)}$ is a constant. Thus, it is easy to obtain the general form of $\omega_{z}$ as

$$
\omega_{z}(r, \theta)=\sum_{n=1}^{\infty}\left[a_{\omega, n} r^{n}+b_{\omega, n} r^{-n}+C_{n}^{(2)} \xi^{2} K_{n}\left(\frac{r}{\bar{\xi}}\right)\right] \sin n \theta
$$

It is obvious that $a_{\omega, n}=0$ for all $n$ to satisfy the boundary condition far from the cylinder. From the last equation of (81) we obtain

$$
b_{\omega, n}=-C_{n}^{(2)} K_{n}\left(\xi^{-1}\right) \xi^{2} .
$$

Similarly, we can obtain $\Psi$ as

$$
\begin{aligned}
\Psi(r, \theta)= & \sin \theta\left[a_{\Psi}^{(2)} r+\frac{b_{\Psi}^{(2)}}{r}+\alpha r \ln r-\mu_{r} \xi^{4} C_{1}^{(2)} K_{1}\left(\frac{r}{\xi}\right)\right] \\
& +\sum_{n=2}^{\infty} \sin n \theta\left[\frac{b_{\Psi, n}^{(2)}}{r^{n}}+d_{\Psi, n}^{(2)} r^{2-n}\right. \\
& \left.-\mu_{r} C_{n}^{(2)} \xi^{4} K_{n}\left(\frac{r}{\xi}\right)\right] .
\end{aligned}
$$

As in the case of the flow past a sphere, it is easy to show that the mode $n \geqslant 2$ will become zero. Thus, the problem can be simplified. From Eq. (80) and the first two relations of Eq. (81) we obtain

$$
b_{\omega}=-\alpha, \quad a_{\Psi}^{(2)}=\alpha \hat{a}, \quad b_{\Psi}^{(2)}=\alpha \hat{b}, \quad C_{1}^{(2)}=\frac{\alpha}{\xi^{2} K_{1}\left(\xi^{-1}\right)}
$$

where

$$
\hat{a}=\frac{1}{2}\left[\mu_{r} \xi\left(\xi+\frac{K_{1}^{\prime}\left(\xi^{-1}\right)}{K_{1}\left(\xi^{-1}\right)}\right)-1\right]
$$

$$
\hat{b}=\frac{1}{2}\left[\mu_{r} \xi\left(\xi-\frac{K_{1}^{\prime}\left(\xi^{-1}\right)}{K_{1}\left(\xi^{-1}\right)}\right)+1\right]
$$

Thus, we obtain

$$
\begin{gathered}
\Psi(r, \theta)=\alpha \sin \theta\left(\hat{a} r+\frac{\hat{b}}{r}+r \ln r-\mu_{r} \xi^{2} \frac{K_{1}(r / \xi)}{K_{1}\left(\xi^{-1}\right)}\right), \\
\omega(r, \theta)=\alpha \sin \theta\left(-\frac{1}{r}+\xi^{2} \frac{K_{1}(r / \xi)}{K_{1}\left(\xi^{-1}\right)}\right) .
\end{gathered}
$$

LIke the usual Stokes problem in 2D, the flow field produced from Eq. (88) has a logarithmic singularity in the limit of $r$ $\rightarrow \infty$.

\section{B. The outer solution and matched asymptotic method}

To resolve Stokes' paradox for $r \rightarrow \infty$, we adopt the matched asymptotic method developed by Kaplun and Lagerstrom [23]. We reconsider Eq. (88),

$$
\Psi(r, \theta) \sim \alpha(R)\left(\hat{a} r+\frac{\hat{b}}{r}+r \ln r-\mu_{r} \xi^{2} \frac{K_{1}(r / \xi)}{K_{1}\left(\xi^{-1}\right)}\right) \sin \theta,
$$

where $\alpha$ is replaced by a multiplier $\alpha(R)$ which is allowed to depend upon the Reynolds number, because our asymptotic sequence is unspecified. Although this approximation cannot satisfy the condition $\mathbf{v}=\mathbf{e}_{x}$ in Eq. (40) or $\Psi \rightarrow r \sin \theta$ for $r$ $\rightarrow \infty$, it can be matched to the uniform stream, regarded as the first term of an Oseen expansion.

Now, introducing a new variable

$$
\rho=\frac{R}{1-\mu_{r} / 2} r=\epsilon r ; \quad \epsilon=\frac{R}{1-\mu_{r} / 2},
$$

the outer equation (24) is

$$
\frac{\partial \mathbf{u}}{\partial \hat{x}}=-\hat{\nabla} \tilde{p}+\hat{\Delta} \mathbf{u}
$$

where $\hat{\boldsymbol{\nabla}}=\left(1-\mu_{r} / 2\right)^{-1} \widetilde{\boldsymbol{\nabla}}$. Then the Oseen expansion begins with

$$
\Psi \sim \frac{1}{\epsilon} \rho \sin \theta+\cdots \quad \text { as } \epsilon \rightarrow 0 .
$$

Writing the Stokes expression (89) with the Oseen variable (90), the leading term is now

$$
\Psi \sim \frac{\alpha(\epsilon)}{\epsilon} \ln (1 / \epsilon) \rho \sin \theta,
$$

where $\alpha(\epsilon)=\alpha(R)$ in the limit of $\epsilon \rightarrow 0$. This matches Eq. (92) if $\alpha(\epsilon)=[\ln (1 / \epsilon)+k]^{-1}$ where $k$ is a constant to be determined later.

Expansion of the Stokes approximation (89) further by $\rho$ and $\alpha(\epsilon)$ leads to 
PRE $\underline{61}$

$$
\Psi \sim \frac{1}{\epsilon}[1+\alpha(\epsilon)(\ln \rho-k+\hat{a})] \rho \sin \theta
$$

This requires the Oseen expansion (92) to continue as

$$
\Psi \sim \frac{1}{\epsilon}[\rho \sin \theta+\alpha(\epsilon) \psi(\rho, \theta)+\cdots]
$$

Substituting this into the full equation, $\psi$ satisfies the linearized Oseen equation

$$
\left(\hat{\Delta}-\frac{\partial}{\partial \hat{x}}\right) \hat{\Delta} \psi=0
$$

The appropriate solution for the stream function can be found as an infinite series [63].

The fundamental solution due to Oseen gives as the Cartesian velocity components

$$
\begin{aligned}
u_{x}= & \frac{\partial \psi}{\partial(\rho \sin \theta)} \\
= & 2 c_{2}\left(\frac{\partial}{\partial(\rho \cos \theta)}\left[\ln \rho+e^{(\rho \cos \theta) / 2} K_{0}(\rho / 2)\right]\right. \\
& \left.-e^{(\rho \cos \theta) / 2} K_{0}(\rho / 2)\right), \\
u_{y}= & -\frac{\partial \psi}{\partial(\rho \cos \theta)} \\
= & 2 c_{2} \frac{\partial}{\partial(\rho \sin \theta)}\left[\ln \rho+e^{(\rho \cos \theta) / 2} K_{0}(\rho / 2)\right],
\end{aligned}
$$

where $c_{2}$ is a constant which will be determined by the matching. The term in $\ln \rho$ at the origin cancels the term involving $K_{0}(\rho / 2)$. For small $\rho$ we obtain the integrated form of $\psi$ as

$$
\psi \sim-c_{2}\left(\ln \frac{4}{\rho}+1-\gamma\right) \rho \sin \theta+O\left(\rho^{2} \ln \rho\right),
$$

where $\gamma$ is Euler's constant $\gamma=0.5772 \ldots$. Using this we find that the Oseen expansion (95) behaves near the cylinder as

$$
\Psi \sim \frac{1}{\epsilon} \rho \sin \theta\left[1+c_{2} \alpha(\epsilon)\left(\ln \frac{\rho}{4}+\gamma-1\right)\right] .
$$

This can match Eq. (94) if we choose

$$
c_{2}=1 ; \quad k=\hat{a}-\gamma+1+\ln 4
$$

Thus, we obtain

$$
\alpha(\epsilon)=\left(\ln \frac{4}{\epsilon}-\gamma+1+\hat{a}\right)^{-1}
$$

This vanishes for $\epsilon \rightarrow 0$. The explicit expression near the cylinder is thus given by

$$
\Psi(r, \theta) \simeq \alpha(\epsilon)\left(\hat{a} r+\frac{\hat{b}}{r}+r \ln r-\mu_{r} \xi^{2} \frac{K_{1}(r / \xi)}{K_{1}\left(\xi^{-1}\right)}\right) \sin \theta
$$

This expression satisfies all the boundary conditions.

The explicit expression of the inner solution is thus given by

$$
\begin{gathered}
v_{r}=\alpha(\epsilon)\left(\hat{a}+\frac{\hat{b}}{r^{2}}+\ln r-\mu_{r} \xi^{2} \frac{K_{1}(r / \xi)}{r K_{1}\left(\xi^{-1}\right)}\right) \cos \theta, \\
v_{\theta}=\alpha(\epsilon)\left(-\hat{a}+\frac{\hat{b}}{r^{2}}-\ln r-1+\mu_{r} \xi \frac{K_{1}^{\prime}(r / \xi)}{K_{1}\left(\xi^{-1}\right)}\right) \sin \theta .
\end{gathered}
$$

The pressure is similarly determined from Eqs. (25) and (76) as

$$
\frac{\partial p}{\partial r}=\frac{1}{r} \frac{\partial}{\partial \theta}\left(\Delta \Psi+\mu_{r} \omega_{z}\right), \quad \frac{1}{r} \frac{\partial p}{\partial \theta}=-\frac{\partial}{\partial r}\left(\Delta \Psi+\mu_{r} \omega_{z}\right) .
$$

As in the case of $3 \mathrm{D}$, the pressure is determined by the Stokes pole $\Psi \sim r \ln r$. The result is

$$
p=p_{0}-\frac{2-\mu_{r}}{r} \alpha(\epsilon) \cos \theta
$$

where $p_{0}$ is an unimportant constant. Thus, explicit representations for the inner solution, which can be connected with the outer solution, are obtained.

\section{Drag force exerted on a cylinder}

As in the previous section, let us calculate the drag force exerted on a cylinder by a moving fluid. The drag force is again calculated from three contributions. The force coming from the asymmetric tensor is not zero in 2D. From Eq. (67), the result is

$$
F_{\tau}=-\left.\mu_{r} \int_{0}^{2 \pi y} \frac{y}{r} \Omega(1, \theta)\right|_{r=1} d \theta=-\mu_{r} \int_{0}^{2 \pi} \Omega(1, \theta) \sin \theta d \theta
$$

where $\Omega=(\operatorname{rot} \mathbf{v})_{z} / 2$ and it is reduced to $\Omega(1, \theta)=\frac{1}{2} \partial v_{\theta} / \partial r$. From Eq. (87) and (103) we obtain

$$
F_{\tau}=\pi \mu_{r} \alpha(\epsilon)\left[1-\mu_{r} \beta(\epsilon)\right]
$$

where we use $\int_{0}^{2 \pi} \sin ^{2} \theta=\pi$ and

$$
\beta(\xi)=\frac{1}{2 K_{1}\left(\xi^{-1}\right)}\left[K_{1}^{\prime \prime}\left(\xi^{-1}\right)-\xi K_{1}^{\prime}\left(\xi^{-1}\right)+\xi^{2} K_{1}\left(\xi^{-1}\right)\right]
$$

The force coming from the normal stress is given by

$$
F_{p}=-\int_{0}^{2 \pi} p \cos \theta d \theta=\pi\left(2-\mu_{r}\right) \alpha(\epsilon)
$$

and the force from the shear stress is 


$$
\begin{aligned}
F_{f} & =-\left.\left(1-\frac{\mu_{r}}{2}\right) \int_{0}^{2 \pi} d \theta \sin \theta \frac{\partial v_{\theta}}{\partial r}\right|_{r=1} \\
& =\pi\left(2-\mu_{r}\right) \alpha(\epsilon)\left[1-\mu_{r} \beta(\xi)\right] .
\end{aligned}
$$

Thus, the total force is given by

$$
F_{t o t}=\pi \alpha(\epsilon)\left[4-\mu_{r}-2 \mu_{r} \beta(\xi)\right]
$$

which can be rewritten as

$$
F_{t o t}=\pi\left(\eta+\eta_{r}\right) U \alpha(\epsilon)\left[4-\mu_{r}-2 \mu_{r} \beta(\xi)\right]
$$

in physical units. This is reduced to the result obtained from the Oseen approximation [64] as $F_{\text {tot }} \rightarrow 4 \pi \eta U /(S+1 / 2)$ in the limit of $\mu_{r} \rightarrow 0$ where $S=\ln (4 / R)-\gamma$.

\section{STEADY FLOW INSIDE A CONTAINER}

In this section we discuss steady circulation flows inside a container. Since the flow is confined in a finite region, the Stokes approximation may give an appropriate solution for the slow viscous flow. Thus, the basic equations in this section are Eqs. (25) and (26).

As mentioned in Sec. III, we do not impose the boundary condition $\mathbf{v}=0$ but the slip boundary condition (15) on the surface of the container, because otherwise the problem becomes overcomplete. This may be understood from the analogy of granular flows as follows. The rotation of particles without the slip boundary produces a fast flow near the boundary. The width of the boundary layer is as thin as the particle radius. If we are able to normalize the flow by the fastest flow rate near the boundary, we may be able to solve the problem under the condition $\mathbf{v}=0$ on the wall. It is difficult, however, to determine the position where the flow rate is maximum theoretically, and the width of the boundary layer becomes zero as the radius of the particles becomes zero. Thus, we adopt the slip boundary condition in this section.

We note that the actual situation needs an external force to maintain the flow. If we regard this flow as an approximate one for vibrating beds, there is external vibration and stationary gravity. If we assume that the time dependence of the pressure, the velocity, and the microrotation are represented by a common function $\Xi(t)$, e.g., $\Xi(t)=1-\Gamma \cos t$, Eq. (25) is now replaced by

$$
\Xi(t)\left[-\nabla(-z+p)-\mathbf{e}_{z}+\Delta \mathbf{v}+\mu_{r} \operatorname{rot}\left(\omega \mathbf{e}_{\perp}\right)\right]=0,
$$

where the gravity $-\mathbf{e}_{z}$ and the stationary pressure $-z$ cancel each other. Since the time dependence and the effect of gravity in such a linear system can be absorbed in $\Xi(t)$, we can discuss the flow inside the container as a stationary problem.

\section{A. Flow inside a cylinder}

Let us demonstrate the existence of an interesting circulation flow inside the cylinder. The surface of a micropolar fluid exists at $z=0$ and $z$ increases as depth from the surface increases. Let us consider the flow $\mathbf{v}=\left(v_{r}, v_{\theta}, v_{z}\right)$ $=\left(v_{r}(r, z), 0, v_{z}(r, z)\right)$. In this case the flow can be represented by a vector potential or a stream function $\mathbf{v}$ $=\operatorname{rot}\left[\Psi(r, z) \mathbf{e}_{\theta}\right]$ or

$$
v_{r}=-\frac{\partial \Psi}{\partial z} ; \quad v_{z}=\frac{1}{r} \frac{\partial}{\partial r}(r \Psi) .
$$

Since the microrotation field is represented by $\boldsymbol{\omega}=\omega_{\theta} \mathbf{e}_{\theta}$, the basic equations (25) and (26) can be rewritten as

$$
\mathcal{D}^{4} \Psi+\mu_{r} \mathcal{D}^{2} \omega_{\theta}=0
$$

and

$$
\mathcal{D}^{2}\left(\mathcal{D}^{2}-\xi^{-2}\right) \omega_{\theta}=0,
$$

where $\mathcal{D}^{2}$ is defined by

$$
\mathcal{D}^{2}=\frac{\partial^{2}}{\partial r^{2}}+\frac{1}{r} \frac{\partial}{\partial r}-\frac{1}{r^{2}}+\frac{\partial^{2}}{\partial z^{2}}
$$

The boundary condition (15) and the conservation law (16) are reduced to

$$
\Psi(1, z)=\omega_{\theta}(1, z)=0, \quad \frac{\partial \Psi}{\partial z}=\mathcal{D}^{2} \Psi=\omega_{\theta}=0 \quad \text { at } \quad r=0
$$

Unfortunately, the conditions at the center of cylinder will be identities, which will not be useful to determine unknown constants.

Assuming $\mathcal{D}^{2} \omega_{\theta}=R_{\theta}(r) Z(z)$, Eq. (116) is reduced to

$$
\frac{1}{R_{\theta}}\left(\frac{d^{2}}{d r^{2}}+\frac{1}{r} \frac{d}{d r}-\frac{1}{r^{2}}\right) R_{\theta}=-\frac{1}{Z} \frac{d^{2} Z}{d z^{2}}=-\lambda,
$$

where $\lambda$ is a separation constant. In the standard sense, $\lambda$ should be negative, because a flow exists along the stream line ( $\Psi$ is a constant). In this case the flow becomes a circular flow or a convection. For $\lambda<0, Z$ is expected to be $\lambda$ $=\lambda_{n}=(n / H)^{2}$ with a positive integer $n$, and

$$
Z_{n}(z)=\sin (n z / H),
$$

where $H$ is the height of the cylinder. The equation for $R_{\theta}$ is now solvable as $R_{\theta} \sim I_{1}\left[r /(H / n)^{2}\right]$ where $I_{1}(z)$ is the modified Bessel function. Thus we obtain

$$
\mathcal{D}^{2} \omega_{\theta}=\sum_{n=1}^{\infty} c_{\theta, n} I_{1}\left(\frac{r}{(H / n)^{2}}\right) \sin \left(\frac{n z}{H}\right)
$$

where $c_{\theta, n}$ is a constant. For later use, we discuss only the mode $n=1$ because of its fundamental role. From Eq. (121) we obtain

$$
\omega_{\theta}=C_{0}\left[C_{1} I_{1}(r / H)+\xi^{2} I_{1}\left(r / \zeta_{0}\right)\right] \sin \left(\frac{z}{H}\right)
$$

where $C_{0}$ and $C_{1}$ are constants, and $\zeta_{0}=\xi / \sqrt{1+(\xi / H)^{2}}$. From Eq. (115) we obtain 


$$
\Psi=C_{0} \xi^{4} \mu_{r}\left(\frac{I_{1}\left(1 / \zeta_{0}\right)}{I_{1}(1 / H)} I_{1}(r / H)-I_{1}\left(r / \zeta_{0}\right)\right) \sin \left(\frac{z}{H}\right)
$$

where we use the boundary condition (118). Note that we omit a formal special solution of Eq. (115) coming from the first term of Eq. (122), because it becomes singular at the center of the container.

From Eq. (114) the circular flow arising from Eq. (123) is given by

$$
v_{r}=-\frac{1}{H} C_{0} \xi^{4} \mu_{r}\left(\frac{I_{1}\left(1 / \zeta_{0}\right)}{I_{1}(1 / H)} I_{1}(r / H)-I_{1}\left(r / \zeta_{0}\right)\right) \cos \left(\frac{z}{H}\right),
$$

and

$$
v_{z}=C_{0} \xi^{4} \mu_{r}\left(\frac{1}{H} \frac{I_{1}\left(1 / \zeta_{0}\right)}{I_{1}(1 / H)} I_{0}(r / H)-\frac{1}{\zeta_{0}} I_{0}\left(r / \zeta_{0}\right)\right) \sin \left(\frac{z}{H}\right),
$$

where we use $(1 / z)(d / d z)\left[z I_{1}(z)\right]=I_{0}(z)$. Equation (125) is reduced to

$$
v_{z} \simeq C_{0} \xi^{4} \mu_{r}\left[2 I_{1}(1 / \xi)-\xi^{-1} I_{0}(r / \xi)\right] \sin \left(\frac{z}{H}\right)
$$

for $H \gg 1$ [i.e., $\zeta_{0} \rightarrow \xi$ and $\left.I_{1}(1 / H) \sim 1 /(2 H)\right]$. Although Eq. (126) contains a similar $r$ dependence to that observed in experiments on granular particles in vibrating beds [17], the $z$ dependence of the functional form is different.

Let us examine the case of $\lambda>0$. This corresponds to the situation in which the flow is localized near the surface. By a parallel procedure to that of Sec. V, we obtain

$$
\mathcal{D}^{2} \omega_{\theta}=c_{0} I_{1}(r / \zeta) \exp (-\sqrt{\lambda} z)
$$

where $I_{1}(x)$ is the modified Bessel function, $\lambda$ is an undetermined separation constant in Eq. (119) assumed to be $\lambda$ $<1 / \xi^{2}$, and $\zeta=\xi / \sqrt{1-\lambda \xi^{2}}$. Thus, we obtain

$$
\omega_{\theta}=c_{0}\left[c_{1} J_{1}(\sqrt{\lambda} r)+\xi^{2} I_{1}(r / \zeta)\right] \exp (-\sqrt{\lambda} z)
$$

where $J_{1}(z)$ is the Bessel function and $c_{0}$ and $c_{1}$ are constants that are determined by the boundary condition for $\omega_{\theta}$. From Eqs. (115) and (118) we obtain

$$
\Psi(r, z)=c_{0} \xi^{4} \mu_{r}\left(\frac{I_{1}(1 / \zeta)}{J_{1}(\sqrt{\lambda})} J_{1}(\sqrt{\lambda} r)-I_{1}(r / \zeta)\right) \exp (-\sqrt{\lambda} z)
$$

This solution may not be a solution for a smooth flow, because the flow along the stream line ( $\Psi=$ const) cannot circulate as a convection flow. Since the flow in vibrating beds is not smooth, we may examine this solution as a steady solution for granular flows.

The solution of the flow field described by Eq. (129) is given by

$$
v_{r}=c_{0} \xi^{4} \mu_{r} \sqrt{\lambda}\left(\frac{I_{1}(1 / \zeta)}{J_{1}(\sqrt{\lambda})} J_{1}(\sqrt{\lambda} r)-I_{1}(r / \zeta)\right) \exp (-\sqrt{\lambda} z)
$$

and

$$
v_{z}=c_{0} \xi^{4} \mu_{r}\left(\sqrt{\lambda} \frac{I_{1}(1 / \zeta)}{J_{1}(\sqrt{\lambda})} J_{0}(\sqrt{\lambda} r)-\frac{1}{\zeta} I_{0}(r / \zeta)\right) \exp (-\sqrt{\lambda} z)
$$

The result (131) is reduced to

$$
v_{z} \simeq c_{0} \xi^{4} \mu_{r}\left(2 I_{1}(1 / \xi)-\frac{1}{\xi} I_{0}(r / \xi)\right) \exp (-\sqrt{\lambda} z)
$$

in the limit of $\lambda \rightarrow 0$, which is identical to that reported in experiments on vibrating beds [17]. In fact, $v_{z}$ in Eq. (11b) of [17] is given by

$$
v_{z}=\frac{l}{\tau}\left(1+\frac{1}{f_{I}(1 / \xi)}\left[1-I_{0}(r / \xi)\right]\right) e^{-z / l},
$$

where $l$ and $\tau$ are respectively the characteristic length scale and the time scale, and $f_{I}(x)=2 I_{1}(x) / x-1$ from the conservation law (16). Equation (133) can be rewritten as

$$
v_{z} \propto\left[2 \xi I_{1}(1 / \xi)-I_{0}(r / \xi)\right] e^{-\sqrt{\lambda z}}
$$

identifying $l=1 / \sqrt{\lambda}$. Thus, Eq. (134) is identical to Eq. (132). On the other hand, Eq. (130) cannot describe convection flows, because $v_{r}$ in circulation flows must have turning point(s) to change the direction of flow. However, we expect that the flow is similar to that observed in experiments.

\section{B. Flow inside a rectangular container}

For the 2D case it is easy to derive Eq. (11b) of Ref. [17] by a parallel discussion to that presented here. Introducing

$$
v_{x}=\frac{\partial \Psi}{\partial z}, \quad v_{z}=-\frac{\partial \Psi}{\partial x}
$$

we obtain Eqs. (77)-(79). From a similar discussion to that in the $3 \mathrm{D}$ case we immediately obtain

$$
\omega_{z}(x, y)=\left[a_{2 D}+b_{2 D} \sinh (x / l)\right] \exp \left(-\sqrt{\lambda_{2 D}} z\right)
$$

where $a_{2 D}$ and $b_{2 D}$ are constants determined from the boundary conditions, $l=\xi / \sqrt{1-\lambda_{2 D} \xi^{2}}$, and $\lambda_{2 D}$ is the separation constant. Thus, it is straightforward to obtain

$$
\Psi(x, z)=-\left[c_{2 D}+\tilde{a}_{2 D} x+\tilde{b}_{2 D} \sinh (x / l)\right] \exp \left(-\sqrt{\lambda_{2 D}} z\right),
$$

where $c_{2 D}, \tilde{a}_{2 D}$ and $\tilde{b}_{2 D}$ are constants. The velocity field is obtained as

$$
v_{z}=\left(\tilde{a}_{2 D}+\frac{\tilde{b}_{2 D}}{l} \cosh (x / l)\right) \exp \left(-\sqrt{\lambda_{2 D}} z\right)
$$

and

$$
v_{x}=\sqrt{\lambda_{2 D}}\left[\tilde{a}_{2 D} x+\tilde{b}_{2 D} \sinh (x / l)\right] \exp \left(-\sqrt{\lambda_{2 D}} z\right) .
$$

Note that $c_{2 D}=0$ in Eq. (137) because of the symmetry of the flow $\left(v_{x}=0\right)$ at the center of the container $x=0$. The 
result of Eq. (138) is identical to the corresponding equation by Knight et al. [17]. We also indicate that both Eqs. (138) and (139) recover the result of the DEM simulation, though the change of sign of $v_{x}$ as a function of $z$ is not obtained in the above expression [18].

The calculation in this section is not matured when we compare it with those in the previous sections. However, the result is suggestive, and is comparable with experimental observation of vibrating beds of granular flow. Therefore, we can expect the existence of close relationships between micropolar fluids and granular flows. Of course, this agreement may be accidental. In fact, similar results can be reproduced by different models such as the hopping diffusion model [65]. From the critical point of view, we may deduce that the micropolar fluid model is not appropriate for granular flows because the separation constant cannot be determined within this framework. We will need more systematic investigations.

\section{DISCUSSION AND CONCLUSION}

In this paper, we illustrate systematic calculations of the viscous micropolar flows around a cylinder and a sphere, and a preliminary calculation of the steady flows inside a container. If the micropolar fluid is an important concept, the first two calculations will play fundamental roles. On the other hand, the last calculation demonstrates that the solution for the micropolar fluid is identical to that observed in granular flows, though the agreement may be superficial. Although the result itself is only valid for Newtonian micropolar fluids, the agreement of our result with the experiment and simulations of granular flows is attractive for the application of micropolar models to granular flows.

As indicated in Sec. II, micropolar fluid mechanics cannot be a microscopic model but must be a phenomenology. Thus, it is natural to be skeptical of our approach to seek an appropriate effective fluid theory of granular flows. To answer this objection in part, we have demonstrated some points of relevancy of the calculation based on the Newtonian micropolar fluid model. We will compare its result with the result of a DEM simulation in detail elsewhere [18]. However, we note that the comparison is relevant only in the region far from the onset of convection, because below the onset the granular particles are not in a fluidlike but in a solidlike state. As mentioned Sec. IV, we can predict not only the vertical velocity profile but also the horizontal velocity profile. After averaging a large number of cycles (e.g., 1000 in [18]), we can suppress most stagnant effects, such as avalanches of arches and the flow caused by fractures in granular beds. Thus we can obtain a smooth velocity profile as a function of time. The result of our simulation in most time regions supports the relevancy of the micropolar calculation in Sec. VI. Unfortunately, there remains the effect of avalanches of arches in the bottom part of containers. This part cannot be described by our micropolar fluid calculation. We hope that it is worthwhile to check the validity of micropolar fluid mechanics in granular flows.

Coming back to the flow outside the cylinder/sphere, we will have to compare results reported here with simulations and experiments. In particular, almost all experiments and simulations have focused on stick-slip motion under slow shear forces [66-69], and need a solidlike state to describe stationary states under the acting shear force. The existence of a stick-slip region may lead to the suspicion that a simple application of the micropolar fluid model to granular flow cannot be used. Even when the boundary effects are crucial and the flow coexists with a solidlike state, we believe that our calculation is meaningful, because such a situation can be described by a sort of dynamical fluid-solid transition, and the flow part can be described by a relevant fluid model such as the micropolar fluid model. In any case, our paper may be useful to introduce the unfamiliar concept of micropolar fluid mechanics to granular physicists, where in some cases its validity has already been confirmed quantitatively.

As mentioned in the Introduction, our main concern is not direct application of micropolar fluid mechanics to granular flows, though we are afraid that we have stressed this possibility too much. One of the most important characteristics of micropolar fluids is the existence of a relaxation term of the microrotation to the rotation of flow in Eq. (12). This is common in fluid models describing fluidized beds and traffic flows [70-72]. Traffic flows and granular flows in a tube are known to have a beautiful mathematical structure [73-75]. However, there are few theoretical arguments for two dimensional flows. Micropolar fluid mechanics may give a universal framework to discuss higher dimensional flows with microstructure.

In conclusion, we have investigated fundamental properties of slow micropolar fluid flows. We have presented systematic calculation of flows around a sphere and a cylinder. We obtained explicit forms of the velocity fields [Eqs. (61) and (103)], the microrotation fields [Eq. (62)] and the drag force exerted by the fluid flow [Eqs. (72) and (112)]. We also gave the procedure to calculate contributions from singular perturbations of the Reynolds number. We found an interesting solution (132) for a steady flow inside a cylinder, which is identical to Eq. (133) observed in experiments on granular vibrating beds.

\section{ACKNOWLEDGMENTS}

The author thanks T. Ayukawa and A. Shimosaka for useful discussions. This work was partially supported by a Grant-in-Aid for Science Research from the Ministry of Education, Science and Culture (Grant No. 11740228).
[1] E. Cosserat and F. Cosserat, Theòrie des Corps Dèformables (A. Hermann, Paris, 1909).

[2] D.W. Condiff and J.S. Dahler, Phys. Fluids 7, 842 (1964).

[3] A.C. Eringen, J. Math. Mech. 16, 1 (1966).

[4] G. Lukaszewicz, Micropolar Fluids: Theory and Applications
(Birkhäuser, Boston, 1999).

[5] M.I. Shliomis, Zh. Éksp. Teor. Fiz. 51, 258 (1967) [Sov. Phys. JETP 24, 173 (1967)]; see also R. E. Rosensweig, Ferrohydrodynamics (Cambridge University Press, Cambridge, 1985).

[6] M.A. Turk, N.D. Syvester, and T. Ariman, Trans. Soc. Rheol. 
17, 1 (1973).

[7] G. Bugliarello and J. Sevila, J. Biorheol., 7, 85 (1970).

[8] A.C. Eringen, Int. J. Eng. Sci. 28, 133 (1990).

[9] See, for example, P.G. de Gennes and J. Prost, The Physics of Liquid Crystals, 2nd ed. (Oxford University Press, Oxford, 1993).

[10] H.M. Jaeger, S.R. Nagel, and R.B. Behringer, Rev. Mod. Phys. 68, 1259 (1996).

[11] Physics of Dry Granular Media, edited by H.J. Herrmann, J.-P. Havi, and S. Luding (Kluwer, Dordrecht, 1998).

[12] Powders and Grains 97, edited by R.P. Behringer and J.T. Jenkins (A.A. Balkema Pub., Rotterdam, 1997).

[13] H. Hayakawa, H. Nishimori, S. Sasa, and Y.-H. Taguchi, Jpn. J. Appl. Phys., Part 1 34, 397 (1995); Physics of Granular Matter, edited by H. Nishimori, H. Hayakawa, and Y.-H. Taguchi, Selected Papers in Physics Vol. XI, (The Physical Society of Japan, Tokyo, 1999). Note that the review parts of the latter book are written in Japanese.

[14] Granular Matter, edited by A. Mehta (Springer, Berlin, 1994).

[15] K. Kanatani, Trans. Jpn. Soc. Mech. Eng., Ser. B 45, 507 (1979); 45, 515 (1979).

[16] J. Kano, A. Shimosaka, and J. Hidaka, J. Soc. Powder Technol. Jpn. 33, 95 (1996).

[17] J.B. Knight, E.E. Ehriche, V.Yu. Kupermann, J.K. Flint, H.M. Jaeger, and S.R. Nagel, Phys. Rev. E 54, 5726 (1996).

[18] S. Wada and H. Hayakawa, (unpublished).

[19] H. Ramkissoon, Z. Angew. Math. Mech. 65, 635 (1985).

[20] H. Power and H. Ramkissoon, Math. Methods Appl. Sci. 17, 1115 (1994).

[21] T. Buchukuri and R. Chichinadze, Georgian Math J. 1, 251 (1994).

[22] S. Kaplun, J. Math. Mech. 6, 595 (1957).

[23] S. Kaplun and P.A. Lagerstrom, J. Math. Mech. 6, 515 (1957).

[24] P.A. Lagerstrom, J. Math. Mech. 6, 605 (1957).

[25] M. Van Dyke, Perturbation Methods in Fluid Mechanics (Parabolic Press, Stanford, 1975).

[26] J. Kevorkian and J. D. Cole, Multiple Scale and Singular Perturbation Methods (Springer, Berlin, 1996).

[27] P.A. Cundall and O.D.L. Strack, Geotechnique 29, 47 (1979).

[28] For example, for nontriviality of inelastic collisions, see W.A. Morgado and I. Oppenheim, Phys. Rev. E 55, 1940 (1997); N.V. Brilliantov, F. Spahn, J.-F. Hertzch, and T. Pöschel, ibid. 53, 5382 (1996); F. Gerl and A. Zippelius, ibid. 59, 2361 (1999).

[29] I. Goldhirsch and G. Zanetti, Phys. Rev. Lett. 70, 1619 (1993).

[30] D. Enskog, K. Sven. Vetenskapsakad. Handl. 63, 4 (1922); see also J. A. McLennan, Introduction to Non-Equilibrium Statistical Mechanics (Prentice-Hall, Englewood Cliffs, NJ, 1989).

[31] C.S. Campbell, Annu. Rev. Fluid Mech. 22, 57 (1990).

[32] S.B. Savage and D.J. Jeffrey, J. Fluid Mech. 110, 255 (1981).

[33] See, e.g., Recent Developments on Debris Flows, Vol. 64 of Lecture Notes in Earth Sciences, edited by A. Armanini and M. Michiue (Springer, Berlin, 1997).

[34] J.T. Jenkins, and S.B. Savage, J. Fluid Mech. 130, 187 (1983).

[35] C.K.K. Lun, S.B. Savage, D.J. Jeffrey, and N. Chepurniy, J. Fluid Mech. 140, 223 (1984).

[36] J.T. Jenkins and M.W. Richman, Phys. Fluids 28, 3485 (1985).

[37] S.B. Savage and M. Sayed, J. Fluid Mech. 142, 391 (1984).

[38] P.K. Haff, J. Fluid Mech. 134, 401 (1983).

[39] Y. Du, H. Li, and L. Kadanoff, Phys. Rev. Lett. 74, 1268 (1995).
[40] S. McNamara and W.R. Young, Phys. Fluids A 5, 34 (1993).

[41] N. Sela and I. Goldhirsch, Phys. Fluids 7, 507 (1995).

[42] J.J. Brey, F. Moreno, and J.W. Dufty, Phys. Rev. E 54, 445 (1996); J.J. Brey, M.J. Ruitz-Montero, and D. Cubero, ibid. 54, 3664 (1996).

[43] M. Bourzutschky and J. Miller, Phys. Rev. Lett. 74, 2216 (1994).

[44] H. Hayakawa, S. Yue, and D.C. Hong, Phys. Rev. Lett. 75, 2328 (1995).

[45] H. Hayakawa and D. C. Hong, in Powders and Grains 97 (Ref. [12]), p. 451.

[46] D.C. Hong and S. Yue , Phys. Rev. E 58, 4763 (1998).

[47] A. Mehta, Physica A 186, 121 (1992); G.C. Baker and A. Mehta, Nature (London) 364, 486 (1993).

[48] H. Hayakawa and D.C. Hong, Phys. Rev. Lett. 78, 2764 (1997).

[49] M.A. Goodman and S.C. Cowin, J. Fluid Mech. 45, 321 (1971).

[50] S.L. Soo, G.J. Trezek, R.C. Dimick, and G.F. Hohnstreiter, Ind. Eng. Chem. Fundam. 3, 98 (1964).

[51] T.G. Drake, J. Fluid Mech. 225, 121 (1991); J. Geophys. Res. 95, 8681 (1990).

[52] H.A. Janssen, VFDB Z. 39, 1045 (1895). Unfortunately, Janssen's argument is not self-consistent. As a result, most textbooks contain a misunderstanding of the critical stress state. For example, it is possible to show that the principal axis is curved near the sidewall. Thus, all the arguments based on wave equations are wrong.

[53] S.F. Edwards, in Granular Matter (Ref. [14]).

[54] S.F. Edwards and D.V. Grinev, Phys. Rev. Lett. 82, 5397 (1999); Physica A 263, 545 (1999).

[55] T.G. Mason, A.J. Levine, D. Etras, and T.C. Halsey, Phys. Rev. E 60, 5044 (1999).

[56] D.M. Walker, Chem. Eng. Sci. 21, 975 (1966); J.K. Walters, ibid. 28, 13 (1973) These papers clearly state the curve of the principal axis due to the existence of walls. The curve of the axis is easily derived from the assumption of the standard critical stress state.

[57] S.F. Edwards and C.C. Mounfield Physica A 226, 257 (1996).

[58] J.P. Wittmer, P. Claudin, M.E. Cates, and J.-P. Bouchaud, Nature (London) 382, 336 (1996); J.P. Wittmer, M.E. Cates, and P. Claudin J. Phys. I 7, 39 (1997). References [57,58] assume fixed principal axes but the assumption may not be needed, because the axes are curved near the boundary.

[59] S.B. Savage, in Powders and Grains 97 (Ref. [12]), p. 185.

[60] N.P. Migun and P.P. Prohorenko, Hydrodynamics and Heat Exchange for Gradient Flows of Fluid with Microstructure (Minsk, 1984) (in Russian).

[61] C.S. Campbell, J. Fluid Mech. 247, 137 (1993).

[62] G. Peng and T. Ohta, Phys. Rev. E 55, 6811 (1997).

[63] I. Proudman and J.R.A. Pearson, J. Fluid Mech. 2, 237 (1957).

[64] See, e.g., I. Imai, Proc. R. Soc. London, Ser. A 224, 141 (1954).

[65] T. Shinbrot, D. Khakhar, J.J. McCarthy, and J.M. Ottino, Phys. Rev. Lett. 79, 829 (1997).

[66] P.A. Thompson and G.S. Grest, Phys. Rev. Lett. 67, 1151 (1991).

[67] S. Nasuno, A. Kudrolli, and J.P. Gollub, Phys. Rev. Lett. 79, 949 (1997); S. Nasuno, A. Kudrolli, A. Bak, and J.P. Gollub, Phys. Rev. E 58, 2161 (1998).

[68] J.M. Carlson and A.A. Batista, Phys. Rev. E 53, 4153 (1996); 
A.A. Batista and J.M. Carlson, ibid. 57, 4986 (1998).

[69] H. Hayakawa, Phys. Rev. E 60, 4500 (1999).

[70] G.K. Batchelor, J. Fluid Mech. 193, 75 (1988).

[71] S. Sasa and H. Hayakawa, Europhys. Lett. 17, 685 (1992).

[72] B.S. Kerner and P. Konhäuser, Phys. Rev. E 48, 2335 (1993); 50, 54 (1994).
[73] O. Moriyama, N. Kuroiwa, M. Matsushita, and H. Hayakawa, Phys. Rev. Lett. 80, 2833 (1998).

[74] H. Hayakawa and K. Nakanishi, Phys. Rev. E 57, 3839 (1998); Prog. Theor. Phys. Suppl. 130, 57 (1998).

[75] S. Wada and H. Hayakawa, J. Phys. Soc. Jpn. 67, 763 (1998). 